\title{
Preparation and Characterization of Nitrendipine- loaded Eudragit RL 100 Microspheres Prepared by an Emulsion-Solvent Evaporation Method
}

\author{
SK Basu', R Adhiyaman ${ }^{2 *}$ \\ ${ }^{1}$ Division of Pharmaceutics, Department of Pharmaceutical Technology, Jadavpur University, Kolkata, India. \\ ${ }^{2}$ School of Pharmacy and Health Sciences, International Medical University, Kuala Lumpur, Malaysia.
}

\begin{abstract}
Purpose: The aim of the work was to prepare nitrendipne-loaded Eudragit RL 100 microspheres to achieve sustained release nitrendipine.

Method: Nitrendipne-loaded Eudragit RL 100 microspheres were prepared by an emulsion-solvent evaporation method using ethanol/liquid paraffin system. The resultant microspheres were evaluated for average particle size, drug loading, in vitro drug release and release kinetics. FTIR spectrometry, scanning electron microscopy, differential scanning calorimetry and $x$-ray powder diffractometry were used to investigate the physical state of the drug in the microspheres.

Result: The mean particle size of the microspheres was influenced by varying drug:polymer ratio and emulsifier concentration while drug loading was dependent on drug:polymer ratio. The results of FTIR spectrometry, differential scanning calorimetry and $x$-ray diffractometry indicated the stable character of nitrendipne in drug-loaded microspheres and also revealed absence of drug-polymer interaction. The drug release profiles of the microspheres at $\mathrm{pH} 1.2$ showed poor drug release characteristics while at $\mathrm{pH}$ 6.8, drug release was extended over a period of $8 \mathrm{~h}$; release was influenced by polymer concentration and particle size. Drug release followed the Higuchi model.

Conclusion: The nitrendipine-loaded Eudragit RL 100 microspheres prepared under optimized conditions showed a good sustained release characteristics and were stable under the conditions studied.
\end{abstract}

Key words: Nitrendipine, Eudragit RL 100, Microspheres, Physicochemical characterization, Sustained release.

*Corresponding author: Email: genomic2002@yahoo.com; Fax: 0060-86567229 


\section{INTRODUCTION}

Microspheres are one of the particulate delivery systems used to achieve sustained or controlled drug delivery, improve bioavailability and stability and target drug to specific sites. Microspheres also offer advantages such as limiting fluctuation within a therapeutic range, reduction in side effects, decreased dose frequency and hence improved patient compliance ${ }^{1,2}$. The popular method for the encapsulation of drugs within water-insoluble polymers is the emulsion solvent evaporation method. This technique offers several advantages and is preferable to other preparation methods such as spray drying, sonication and homogenization because it requires only mild conditions such as ambient temperature and constant stirring. Thus, a stable emulsion can be formed without compromising the activity of the drugs. The Eudragits are biocompatible copolymers synthesized from acrylic and methacrylic acid esters. These polymers are well tolerated by the skin and have been used in the formulation of dosage forms especially matrix tablets for oral sustained release ${ }^{3,4,5}$ and in tablet coating 6 . They have also been used in the microencapsulation of drugs ${ }^{7,8,9}$. Eudragit $R L$ is insoluble in, but permeable to water and digestive juices, releasing drug by diffusion. Nitrendipine is an orally absorbed calcium antagonist with a short half-life of less than 3 hour; the usual oral dosage regimen is $20 \mathrm{mg}$ to be taken 2 to 3 times a day. Thus nitrendipine is a suitable candidate for oral sustained drug delivery. Therefore, the present study was undertaken to prepare sustained release microspheres of nitrendipne using Eudragit RL 100 by the emulsion solvent evaporation method. The factors affecting particle size, drug loading and drug release behavior of the microspheres were investigated. The physical state of the nitrendipne was also studied using FTIR, DSC, and X-ray diffractrometry.

\section{EXPERIMENTAL Materials}

The following materials were obtained from the indicated suppliers and used as received:
Eudragit RL 100 (Röhm GmbH \& Co. KG, Darmstadt, Germany); ethanol (99\%) and Span 80 (SD-Fine Chemical Works, Mumbai, India); liquid paraffin, di-sodium hydrogen phosphate anhydrous, potassium di-hydrogen phosphate, petroleum ether $\left(40-60^{\circ} \mathrm{C}\right)$, hydrochloric acid and acetic acid glacial (Merck, Mumbai, India). nitrendipine B.P. (Concept Pharmaceuticals, Aurangabad, India)

\section{METHODS}

\section{Preparation of nitrendipine microspheres}

The nitrendipine loaded Eudragit RL100 (EURL 100) microspheres were prepared by the emulsion solvent evaporation method. In this procedure, required amount of nitrendipine and EURL 100 dissolved in ethanol, was emulsified using light liquid paraffin $(80 \mathrm{~mL})$ containing the emulsifier, Span $80(2 \% \mathrm{v} / \mathrm{v})$. The system was stirred continuously using a propeller stirrer at 2000 $\mathrm{rpm}$ and $38 \pm 0.5{ }^{\circ} \mathrm{C}$ for $5 \mathrm{~h}$ to allow the complete evaporation of the solvent. Petroleum ether $\left(40-60{ }^{\circ} \mathrm{C}\right), 100 \mathrm{ml}$, was then added dropwise to the liquid paraffin to harden the microspheres. The paraffin was decanted off, the microspheres were washed repeatedly 4 times with petroleum ether $(10 \mathrm{~mL})$, collected by filtration and finally dried in a hot air oven at $40{ }^{\circ} \mathrm{C}$ for $1 \mathrm{~h}$.

The nitrendipine-loaded EURL microspheres were prepared using varying polymer concentrations $(1,2$ and $3 \% \mathrm{w} / \mathrm{v})$ in the dispersed medium (ethanol, $20 \mathrm{ml}$ ) with varying drug to polymer ratios (i.e., 1:1, 1:2, $1: 3$, and $1: 4$, ) while keeping the other parameter constant. The effects of process variables such as polymer concentration, drug to polymer ratio, and emulsifier concentration on the particle size of the microspheres, drug loading efficiency, and drug release were studied and optimized in the preliminary investigations. The optimum concentration of emulsifier was found to be $2 \%(\mathrm{v} / \mathrm{v})$ and the same concentration was utilized for all the microsphere formulations. 
Table 1: Drug loading, particle size, and in vitro drug release profiles of Nitrendipine - loaded

EURL 100 microspheres prepared at polymer concentrations of $1 \%, 2 \%$ and $3 \% \mathrm{w} / \mathrm{v}$. (Mean \pm S.D.; $n=3$ )

\begin{tabular}{ccccccc}
\hline $\begin{array}{c}\text { Formulation } \\
\text { code }\end{array}$ & $\begin{array}{c}\text { Drug: } \\
\mathbf{R L} \\
\text { ratio } \\
\mathbf{( w / w )}\end{array}$ & $\begin{array}{c}\text { Theoretical } \\
\text { Drug } \\
\text { Content } \mathbf{m} \\
\mathbf{( \% )}\end{array}$ & $\begin{array}{c}\text { Drug } \\
\text { Loading } \\
\mathbf{( \% )} \pm \mathbf{S D}\end{array}$ & $\begin{array}{c}\text { Mean particle } \\
\text { size }(\mu \mathrm{m}) \\
\mathbf{\pm S D}\end{array}$ & $\begin{array}{c}\text { Cumulative \% } \\
\text { drug release } \\
\text { at } \mathbf{p H} 1.2 \text { over } \\
\text { the period of } \\
\text { 4h }\end{array}$ & $\begin{array}{c}\text { Cumulative \% } \\
\text { drug release at } \\
\text { pH } 6.8 \text { over the } \\
\text { period of } 8 \mathrm{~h}\end{array}$ \\
\hline 1\% & & & & & & \\
E1a & $1: 1$ & 50 & $34.46 \pm 0.45$ & $14.25 \pm 6.85$ & $45.73 \pm 1.13$ & $93.69 \pm 0.95$ \\
E1b & $1: 2$ & 33.3 & $23.16 \pm 0.34$ & $16.94 \pm 8.24$ & $43.37 \pm 1.16$ & $90.62 \pm 1.46$ \\
E1c & $1: 3$ & 25 & $18.29 \pm 0.67$ & $19.38 \pm 9.27$ & $41.57 \pm 0.98$ & $85.29 \pm 1.65$ \\
E1d & $1: 4$ & 20 & $15.11 \pm 0.28$ & $21.46 \pm 9.32$ & $40.25 \pm 1.34$ & $85.03 \pm 1.45$ \\
2\% & & & & & & \\
E2a & $1: 1$ & 50 & $36.18 \pm 0.34$ & $24.83 \pm 10.21$ & $43.16 \pm 2.14$ & $89.37 \pm 1.01$ \\
E2b & $1: 2$ & 33.3 & $24.88 \pm 0.39$ & $26.10 \pm 9.87$ & $41.74 \pm 1.82$ & $86.57 \pm 0.84$ \\
E2c & $1: 3$ & 25 & $19.09 \pm 0.75$ & $28.34 \pm 10.28$ & $39.81 \pm 1.86$ & $81.93 \pm 0.48$ \\
E2d & $1: 4$ & 20 & $16.30 \pm 0.29$ & $33.42 \pm 12.16$ & $38.43 \pm 2.18$ & $79.28 \pm 1.47$ \\
3\% & & & & & & \\
E3a & $1: 1$ & 50 & $38.40 \pm 0.44$ & $35.94 \pm 10.85$ & $48.08 \pm 1.64$ & $82.65 \pm 0.45$ \\
E3b & $1: 2$ & 33.3 & $26.53 \pm 1.71$ & $38.56 \pm 11.78$ & $38.29 \pm 1.76$ & $80.66 \pm 0.93$ \\
E3c & $1: 3$ & 25 & $20.61 \pm 0.31$ & $41.28 \pm 12.18$ & $38.46 \pm 0.98$ & $76.97 \pm 1.27$ \\
E3d & $1: 4$ & 20 & $17.27 \pm 0.33$ & $42.15 \pm 12.34$ & $37.59 \pm 0.82$ & $73.40 \pm 0.79$ \\
\hline
\end{tabular}

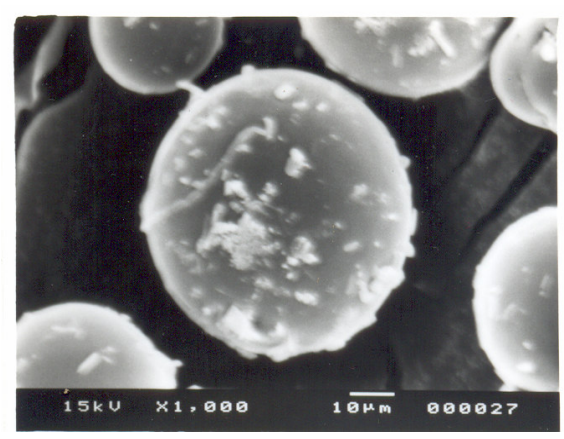

Figure1: Scanning electron micrograph of the nitrendipine-loaded EURL 100 microspheres prepared at $1: 4$ drug: polymer ratio and a polymer concentration of $3 \% \mathrm{w} / \mathrm{v}$.

\section{Particle size analysis and morphological studies}

The mean particle size of the nitrendipine microspheres were determined by optical microscopy. At least 200 microspheres were analyzed for each preparation and the mean diameter was calculated. The surface morphology and appearance of the microspheres were examined by a scanning electron microscopy (JEOL JSM - 5200, Japan) operating between 5-24 kV. The specimens were mounted on a metal stub (with double-sided adhesive tape) and coated under vacuum with gold in nitrogen atmosphere prior to observation. The micrographs are shown in Figure 1.

\section{Determination of drug loading (\%)}

$10 \mathrm{mg}$ of the microspheres was dissolved in a standard flask containing absolute ethanol (10 $\mathrm{ml}$ ) and kept for 12 hours for complete digestion at room temperature. The solution was then filtered through a filter disc (particle 
retention: $11 \mu \mathrm{m})$ and the filtrate was assayed spectrophotometrically for drug content at 356 $\mathrm{nm}$ to compute the drug concentration from the standard curve ${ }^{10,11}$. The drug loading (\%) of the microspheres was calculated using the equations given below.

Drug loading (\%)=M actual $/$ weight of powdered microspheres $\times 100$

where $M_{\text {actual }}$ is the actual drug content in sample of microspheres.

\section{In vitro drug release studies}

Drug-release tests on the microspheres were carried out using a USP dissolution rate test apparatus Type II (Electro Lab model TDT$08 \mathrm{~L})$ for $8 \mathrm{~h}$ at a stirring speed of $100 \mathrm{rpm}$ and temperature of $37 \pm 0.5^{\circ} \mathrm{C}$. An amount of the microspheres equivalent to $20 \mathrm{mg}$ of nitrendipine filled in a hard gelatin capsule (Size no.2) were placed in the dissolution medium containing $600 \mathrm{ml}$ of phosphate buffer (pH 6.8) B.P. containing $0.3 \%(\mathrm{w} / \mathrm{v})$ of sodium dodecyl sulphate (SDS) to maintain sink condition for the drug. A quantity $(5 \mathrm{ml})$ of the dissolution medium was sampled at predetermined time intervals, and fresh dissolution medium was simultaneously used replenish the dissolution medium on each occasion to keep the volume constant. The sample was filtered through filter disc (particle retention: $11 \mu \mathrm{m}$ ), the filtrate diluted to $10 \mathrm{ml}$ with fresh dissolution medium and assayed spectrophotometrically (160 - UV - visible Shimadzu spectrophotometer) at $356 \mathrm{~nm}$ to determine drug concentration. The same procedure was used to assess drug release pattern in hydrochloric acid $(0.1 \mathrm{M})$ buffer solution $(\mathrm{pH}$ 1.2). The release kinetics of nitrendipine from the microspheres was determined using different models, viz, zero order, First order, Higuchi model, PeppasKorsmeyer and Hixon-Crowell. Kinetic assessment of release data was carried out with a program, PCP Disso v2.08 (Anant Ketkar).

\section{FTIR spectroscopic studies}

The IR spectra of the samples were recorded on an FTIR spectrophotometer (Perkin Elmer 1600 series) using $\mathrm{KBr}$ pellet (12 $\mathrm{mm}$ disc), compressed in a hydraulic press at 10 tons for 30 seconds.

\section{Differential scanning calorimetric analysis}

Differential scanning calorimetry (DSC) analysis was undertaken to characterize the changes, if any, during thermal exposure of samples. The test was carried out using a thermal analysis system (Mettler TA 4000 model). Calibration with the standard (indium) was undertaken prior to subjecting the samples for study (between $30-400^{\circ} \mathrm{C}$ ), which were heated at $10^{\circ} \mathrm{C} / \mathrm{min}$ in an aluminum pan under a nitrogen atmosphere while using an empty pan as the reference in this instrument. The instrument automatically calculated onsets of melting point and enthalpy of fusion. The results are shown in Figure 4.

\section{$X$-ray powder diffraction analysis}

The $x$-ray diffraction pattern of the samples were obtained using an $\mathrm{x}$-ray diffractometer (Rich Seifert, model $3000 \mathrm{P}$ ) at $30 \mathrm{kV}, 15 \mathrm{~mA}$ over a range of 10-100 $2 \theta$, using $\mathrm{Cu} \mathrm{Ka}$ radiation wavelength of $1.5405 \mathrm{~A}^{\circ}$. In this technique, the cavity of the metal sample holder of the x-ray diffractometer was filled with ground sample powder and then smoothed out with a spatula. The results are shown in Figure 5.

\section{RESULTS \\ Particle size and morphological characteristics}

Figure 1 shows the scanning electron photomicrograph of the surface of nitrendipine-loaded EURL microspheres having mean particle size $42.15 \pm 12.34 \mu \mathrm{m}$. The particle size of the microspheres increased from $14.25 \pm 6.85$ to $42.15 \pm 12.34$ $\mu \mathrm{m}$ with increasing polymer concentration (Table 1). Few drug crystals appeared on the surface of the microspheres. It was also observed that increasing the emulsifier concentration decreased the microsphere size.

\section{Drug loading of the microspheres}

Increase in the concentration of drug in the organic solvent resulted in an increase in the drug loading of the microspheres. The results indicate that the highest nitrendipine loading 


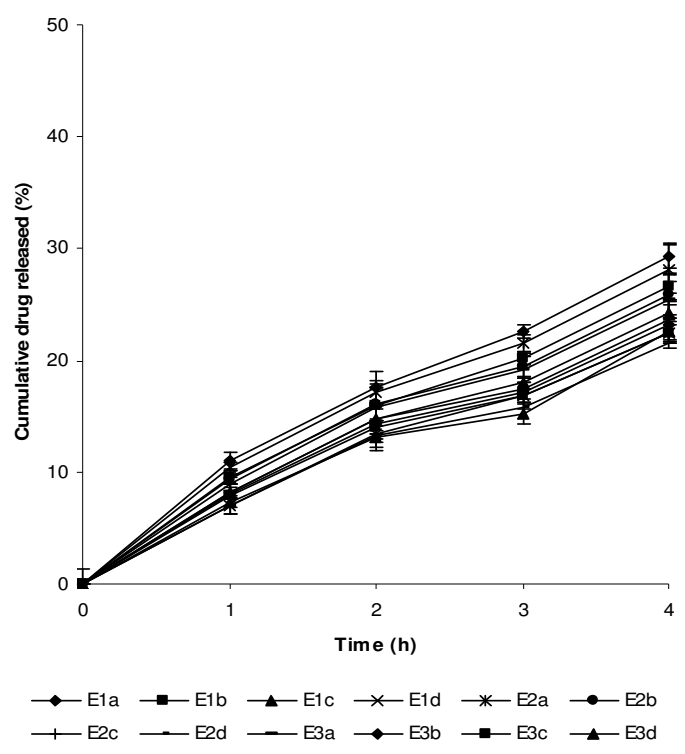

Figure 2: Cumulative drug release of nitrendipine at $\mathrm{pH} 1.2$ hydrochloric acid buffer from nitrendipineloaded Eudragit RL 100 microspheres prepared with 1\% (E1), 2\% (E2) and $3 \%$ (E3) polymer concentrations and at various drug: polymer ratios. (Mean \pm S.D.; $n=3$ ). Note: $a, b, c$ and d represent drug to polymer ratio of $1: 1,1: 2,1: 3$ and 1:4, respectively.

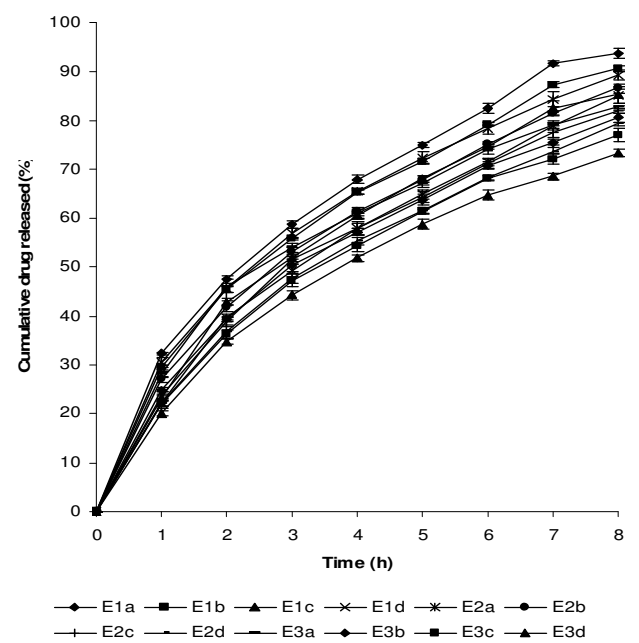

Figure 3: Cumulative drug release of nitrendipine in $\mathrm{pH} 6.8$ phosphate buffer from nitrendipine-loaded Eudragit RL 100 microspheres prepared with 1\% (E1), 2\% (E2) and $3 \%$ (E3) polymer concentrations and at various drug: polymer ratios. Note: $a, b, c$ and $d$ represent drug to polymer ratios 1:1, 1:2, 1:3 and 1:4, respectively.

of $38.40 \pm 0.44 \%$ was observed when the polymer concentration was $3 \%$ at a drug: polymer ratio of $1: 1$ and a stirring rate of 2000 rpm.

\section{In vitro drug release and release kinetic model studies}

Figure 2 indicate that maximum drug release from nitrendipine-loaded microspheres at $\mathrm{pH}$ 1.2 was about $40 \%$ over a period of $4 \mathrm{~h}$. 
Furthermore, there was no significant difference in drug release characteristics of the EURL microspheres irrespective of the polymer concentration and drug: polymer ratio.

Figure 3 illustrates the cumulative percent release of nitrendipine-loaded microspheres at $\mathrm{pH} 6.8$ over a period of $8 \mathrm{~h}$. The data clearly show that drug release (for microspheres prepared at a drug polymer ratio of 1:1) decreased with increase in the polymer concentration as follows: 1, 2, and $3 \%$ polymer concentrations showed $93.69 \pm$ $0.95 \%, 89.37 \pm 1.01 \%, 82.65 \pm 0.45 \%$ drug release, respectively. At other drug : polymer ratios (i.e., 1:2, 1:3, and 1:4) there was only a small retardation of drug release from the microspheres ranging from $86.57 \pm 0.84 \%$ to $73.40 \pm 0.79 \%$ (see Figure 3 ). The EURL microspheres prepared at $3 \%$ polymer concentration showed a certain level of sustained release characteristics, especially microspheres prepared with 1:3 drug to polymer ratio $(\mathrm{E} 3 \mathrm{~d})$ which released $73.40 \pm$ $0.79 \%$ of drug at $\mathrm{pH} 6.8$ over 8 h. An initial 20 $33 \%$ drug release was observed in all the microsphere formulations within the first hour at $\mathrm{pH}$ 6.8. Based on correlation coefficient $\left(r^{2}\right)$ data, the release pattern of nitrendipine from Eudragit $\mathrm{RL}$ microspheres fitted best to the Higuchi model.

\section{FTIR- spectroscopic studies}

FTIR of nitrendipine shows the principal peaks at the wave numbers of $1020.66-1349.17$ $\mathrm{cm}^{-1}$, indicating the presence of carboxyl, carboxylate groups, and carbonyl ester vibrations at $1701.64 \mathrm{~cm}^{-1}$, while $\mathrm{C}-\mathrm{H}$ stretching appeared at $2991.62-2902.96 \mathrm{~cm}$

1 and $\mathrm{NO}_{2}$ group appeared between 1349.17 and $1531.18 \mathrm{~cm}^{-1}$. In the IR spectra of the physical mixture of the formulation ingredients as well as those of nitrendipine-loaded EURL 100 microspheres (E3d), the principal peaks for nitrendipine in the formulation, (E3d), appeared between 1020.66 and $1349.56 \mathrm{~cm}^{-1}$ and indicates the presence of carboxyl, carboxylate groups and carbonyl ester vibration at $1701.74 \mathrm{~cm}^{-1}$, as well as $\mathrm{NO}_{2}$ group between 1349.56 and $1532.34 \mathrm{~cm}^{-1}$.
Therefore, the possibility of any drug polymer interaction is ruled out.

\section{Differential scanning calorimetric studies}

Figure 4 illustrates the DSC thermogram of nitrendipine, physical mixture of nitrendipine with EURL, and nitrendipine-loaded EURL 100 microspheres (E3d). The DSC thermograms of the physical mixture of nitrendipine with Eudragit $R L$, on the one hand, and the microsphere formulation (E3d) on the other, produced almost similar melting endotherms of pure drug at $158.6{ }^{\circ} \mathrm{C}$ and $156.03{ }^{\circ} \mathrm{C}$, respectively. However, the intensity of the drug fusion peak for the microsphere formulation was lower than that of the pure drug and physical mixture.

\section{$X$-ray diffraction studies}

Figure 5 illustrates the comparative X-ray powder diffraction pattern of nitrendipine alone, physical mixture of nitrendipine with EURL, and nitrendipine- loaded EURL microspheres (E3d). The x-ray dffraction profile of Eudragit RL polymer indicated the presence of a completely amorphous material; pure nitrendipine showed the classical diffractogram of the crystalline product. No major difference in the XRD patterns of the physical mixture and the drug loaded microspheres was noticed. However, decreases in the peak intensity and the baseline shift of the diffractogram were observed in the case of the nitrendipineloaded microspheres (E3d) when compared to that of the physical mixture. This is possibly due to the decrease in the degree of crystalinity of the drug following dispersal in the polymer matrix.

\section{DISCUSSION}

Nitrendipine-loaded Eudragit RL microspheres can be easily prepared by solvent evaporation method; the microspheres were satisfactory considering their size and shape. The microsphere size increased with increasing polymer concentration; this may be due to increasing polymer concentration that produced a significant increase in the viscosity, thus leading to an increase of the emulsion droplet size and finally a larger 


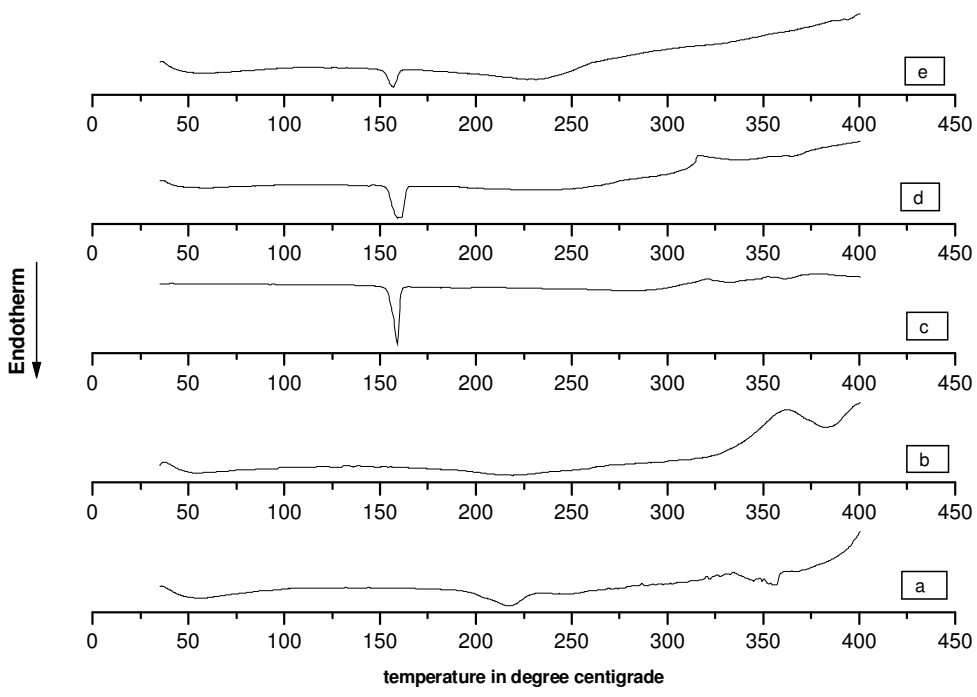

Figure 4: DSC thermograms of Eudragit RL100 (a); Eudragit RL100 microspheres without drug (b); nitrendipine (c); physical mixture of nitrendipine and Eudragit RL100 (d); and nitrendipine-loaded Eudragit RL100 microspheres (e).

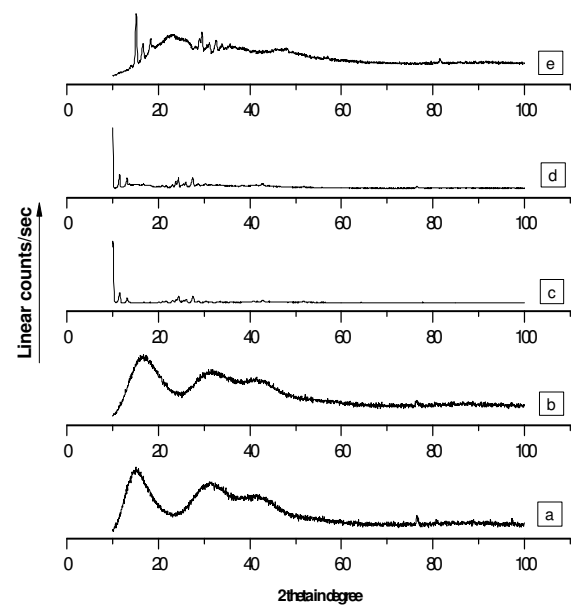

Figure 5: X-ray powder diffraction patterns of Eudragit RL100 (a); Eudragit RL100 microspheres without drug (b); nitrendipine (c); physical mixture of nitrendipine and Eudragit RL100 (d); and nitrendipine-loaded Eudragit RL100 microspheres (e).

microspheres size. On the other hand, microsphere size decreased with increasing emulsifier concentration. The effect of emulsifier concentration on the size of the microspheres can be explained by the higher stability of the dispersed droplets and less tendency of the droplets to coalesce upon collision at higher concentrations of the surfactant. The appearance of drug crystals on the surface of the microspheres may be attributed to high drug concentrations and 
slow solvent removal as the drug formed a particulate (crystal) dispersion resulting.

Drug loading increased with increase in the drug proportion of the preparation. The results of in vitro drug release studies showed that the various drug: polymer ratios and different polymer concentrations had a considerable effect on drug release pattern. The poor drug release and insignificant variation in the drug release pattern of nitrendipine-loaded microspheres at $\mathrm{pH} 1.2$ may be attributed to the effect of the dissolution medium on drug dissolution from the microspheres. The microspheres prepared at a polymer concentration $3 \%$ showed considerable sustained release characteristics, especially microspheres prepared at a 1:3 drug: polymer ratio (E3d) which released $73.40 \pm 0.79 \%$ of drug at $\mathrm{pH} 6.8$ over a period of $8 \mathrm{~h}$ and showed better sustained release characteristics when compared with other microsphere types (Figure 3 ). This may be attributed to the higher polymer content which resulted in a larger particle size and a tightened polymer network and thus retarding drug release. The initial $20-33 \%$ of drug release from all the microspheres may be attributed to the presence of a small proportion of the drug on the microsphere surface which could have arisen from the diffusion of the drug during preparation and drying. When such microspheres are immersed in dissolution medium, the surface drug is immediately released. The assessment of the release kinetics revealed that drug release from the microspheres at $\mathrm{pH}$ 6.8 phosphate buffer followed the Higuchi model. The DSC thermographs of the microspheres showed that the drug fusion peak intensity was less than those for the pure drug and the physical mixture. This may be due to the reduced drug content, (arising from homogeneous dissolution of the drug in the polymer. Other tests indicate that nitrendipine remained in a crystalline state with in the polymer network of the microsphere. It is clearly evident from the XRD pattern, however, that lower peak intensity and a baseline shift were observed for the microsphere when compared to that of the physical mixture. This may be due to a decrease in the degree of crystallinity of the drug.

\section{CONCLUSION}

From the foregoing investigations it was concluded that the nitrendipine loaded Eudragit RL microspheres under optimized conditions showed some degree sustained release and were stable under the conditions studied. The release kinetics followed the Higuchi model.

\section{REFERENCES}

1. Davis SS. The design and evaluation of controlled release systems for the gastrointestinal tract. J. Control. Release 1985; 2: 27-38.

2. Ritschel WA. Biopharmaceutics and Pharmacokinetic aspects in the design of controlled release peroral drug delivery systems. Drug Deve. Ind. Pharm 1989; 15: 1073-1103.

3. Crotts G, Sheth a, Twist J, Sellassie I G. Development of an enteric coating formulation and process for tablets primarily composed of a highly water soluble organic acid. Eur. J. Pharm. Biopharm 2001; 51: 71-76.

4. Nykanen $P$, Lempaa $S$, Aaltonen $M L$, Jurjenson $H$, Veski $P$, Marvola M. Citric acid as excipient in multiple-unit enteric-coated tablets for targeting drugs on the colon. Int. J. pharm 2001; 229: 155-162.

5. Takka S, Rajbhandari S, Saker A. Effect of anionic polymers on the release of propranolol hydrochloride from matrix tablets. Eur. J. Pharm. Biopharm 2001; 52: 75-82.

6. Gupta VK, Beckert TE, Price JC. A novel pH-and timebased multiunit potential colonic drug delivery system. I. Development. Int. J. pharm 2001; 213: 83-91.

7. Lee JH, Park TG Choi HK. Effect of formulation and processing variables on the characteristics of microspheres for water soluble drugs prepared by $w / 0 / 0$ double emulsion solvent diffusion method. Int. J.Pharm 2000; 196: 75-83.

8. Kamel AH, Sokar M.S, Gamal SS, Naggar VF. Preparation and evaluation of ketoprofen coating oral delivery system. Int. J. pharm 2001; 220: 13-21.

9. Perumal D. Microencapsulation ibuprofen and Eudragit RS 100 by the emulsion solvent diffusion technique. Int. J. Pharm 2001;218: 1-11.

10. Kim BK, Hwang SJ, Park JB, Park HJ. Preparation and characterization of drug-loaded polymethacrylate microspheres by an emulsion solvent evaporation method. J. Microencapsul 2002; 19: 811-822.

11. Ming-shi Y, Fu-de C, Ben-gang Y, Yu-ling F, Liang W, Peng $Y, \mathrm{He} Y$. Preparation of sustained-release nitrendipine microspheres with Eudragit RS and 
Basu \& Adhiyaman

Aerosil using quasi-emulsion solvent diffusion method. Int. J. Pharm 2003; 259: 103-113. 